\title{
Inhibitory effects of fasudil on renal interstitial fibrosis induced by unilateral ureteral obstruction
}

\author{
ITSUKO BABA $^{1}$, YASUHIRO EGI ${ }^{1}$, HIROYUKI UTSUMI ${ }^{2}$, TETSUHIRO KAKIMOTO $^{2}$ and KAZUO SUZUKI $^{1}$ \\ ${ }^{1}$ Pharmacology Research Laboratories II; ${ }^{2}$ Safety Research Laboratory, Research Division, \\ Mitsubishi Tanabe Pharma Corporation, Toda-shi, Saitama 335-8505, Japan
}

Received November 27, 2014; Accepted August 25, 2015

DOI: $10.3892 / \mathrm{mmr} .2015 .4467$

\begin{abstract}
Renal fibrosis is the major cause of chronic kidney disease, and the Rho/Rho-associated coiled-coil kinase (ROCK) signaling cascade is involved in the renal fibrotic processes. Several studies have reported that ROCK inhibitors attenuate renal fibrosis. However, the mechanism of this process remains to be fully elucidated. The present study assessed the inhibitory effect of fasudil, a ROCK inhibitor using immunohistochemistry, reverse transcription-quantitative polymerase chain reaction and western blot analyses, in vivo and in vitro, to elucidate the mechanisms underlying renal interstitial fibrosis. In mice induced with unilateral ureteral obstruction (UUO), collagen accumulation, the expression of fibrosis-associated genes and the content of hydroxyproline in the kidney increased 3, 7, and 14 days following UUO. Fasudil attenuated the histological changes, and the production of collagen and extracellular matrix in the UUO kidney. The expression of $\alpha$-smooth muscle actin ( $\alpha$-SMA) and the transforming growth factor- $\beta$ (TGF $\beta$ )-Smad signaling pathway, and macrophage infiltration were suppressed by fasudil in the kidneys of the UUO mice. The present study also evaluated the role of intrinsic renal cells and infiltrated macrophages using NRK-52E, NRK-49F and RAW264.7 cells. The mRNA and protein expression levels of collagen I and $\alpha$-SMA increased in the NRK-52E and NRK-49F cells stimulated by TGF- $\beta 1$. Hydroxyfasudil, a bioactive metabolite of fasudil, attenuated the increase in the mRNA and protein expression levles of $\alpha$-SMA in the two cell types. However, the reduction in the mRNA expression of collagen I was observed in the NRK-49F
\end{abstract}

Correspondence to: Ms. Itsuko Baba, Pharmacology Research Laboratories II, Research Division, Mitsubishi Tanabe Pharma Corporation, 2-2-50 Kawagishi, Toda-shi, Saitama 335-8505, Japan E-mail: baba.itsuko@mk.mt-pharma.co.jp

Abbreviations: ROCK, Rho-associated coiled-coil kinase; UUO, unilateral ureteral obstruction

Key words: unilateral ureteral obstruction, renal interstitial fibrosis, Rho-associated coiled-coil kinase, macrophage infiltration, myofibroblast cells only. Hydroxyfasudil decreased the mRNA expression of monocyte chemoattractant protein-1 (MCP-1) induced by TGF- $\beta 1$ in the NRK-52E cells, but not in the NRK-49F cells. In the RAW264.7 cells, the mRNA expression levels of MCP-1, interleukin (IL)-1 $\beta$, IL-6 and tumor necrosis factor $\alpha$ were increased significantly following lipopolysaccharide stimulation, and were not suppressed by hydroxyfasudil. These data suggested that the inhibition of ROCK activity by fasudil suppressed the transformation of renal intrinsic cells into the myofibroblast cells, and attenuated the infiltration of macrophages, without inhibiting the expression or the activation of cytokine/chemokines, in the progression of renal interstitial fibrosis.

\section{Introduction}

The small G-protein, Rho, and its downstream effector, Rho-associated coiled-coil kinase (ROCK), mediate a variety of cell functions, including smooth muscle contraction, stress fiber formation, cell contraction, adhesion, proliferation, differentiation and inflammatory responses (1-3). The Rho/ROCK signaling pathway is involved in cardiovascular diseases, including hypertension and heart failure, and in chronic kidney disease (4-11).

Fasudil and Y27632, non-selective ROCK1/2 inhibitors, have been used to evaluate the role of ROCK in several animal disease models. For example, in a rat heart ischemia-reperfusion (IR) model, fasudil has been observed to reduce infarct size by attenuating endoplasmic reticulum stress and modulating the activity of sarco/endoplasmic reticulum $\mathrm{Ca} 2^{+}$-ATPase (12). Similarly, in a rat kidney IR model, fasudil has been found to suppress renal injury and improve kidney function (13). Furthermore, fasudil exhibits renoprotective effects, including improving albuminuria and mesangial matrix expansion (14). It also suppresses renal injury via the downregulation of hypoxia-inducible factor $1 \alpha$ in a diabetic nephropathy model (14). Certain in vitro studies, using mesangial, tubular epithelial or kidney fibroblast cells (15-17), have reported that Y27632 inhibits cell hypertrophy, the expression of $\alpha$-smooth muscle actin ( $\alpha$-SMA) and collagen, which is induced by aldosterone or transforming growth factor- $\beta 1$ (TGF- $\beta 1$ ). These reports suggest that ROCK inhibitors use a variety of functional mechanisms and are effective in a wide range of diseases. 
Renal tubulointerstitial fibrosis is a common pathway in progressive renal diseases. The unilateral ureteral obstruction (UUO) model is widely used in investigations of progressive interstitial fibrosis that is independent of hypertension or systemic immune disease. Following UUO, the obstructed kidney exhibits a substantial macrophage influx into the interstitium and develops tubulointerstitial fibrosis. Fasudil and Y27632 have been reported to prevent tubulointerstitial fibrosis in the UUO model by inhibiting the mRNA expression of collagen, TGF- $\beta 1$ and $\alpha$-SMA (18-20). However, the details of the mechanism remain to be fully elucidated. In the present study, the effect of a ROCK inhibitor on renal interstitial fibrosis, renal intrinsic cells and cells infiltrating the kidney were investigated.

\section{Materials and methods}

Animals and ethics. The present study was approved by the Experimental Animal Care and Use Committee of Mitsubishi Tanabe Pharma Corporation (Saitama, Japan), which was regulated by the Management and Ethics of Animal law (Ministry of the Environment; http://www.env.go.jp/nature/dobutsu/aigo/1_ law/). Male C57BL/6 J mice (23-28 g) were purchased from Charles River Laboratories International (Kanagawa, Japan); and were maintained at room temperature on a 12-h light/dark cycle and provided with access to standard laboratory chow (CRF-1, Oriental Yeast Co., Ltd., Tokyo, Japan) and tap water ad libitum. The animals were housed at the departmental animal care facility of Mitsubishi Tanabe Pharma Corporation in accordance with the relevant protocols.

UUO model. The mice were anesthetized with sevofrane (Maruishi Pharmaceutical Co., Ltd., Osaka, Japan) and subjected to a left flank incision. UUO was performed by complete ligation of the left ureter at the ureteropelvic junction using a 4-0 silk suture (cat, no. RS5357; Niccho Kogyo Co., Ltd., Tokyo, Japan). The sham-operated mice had their ureter exposed without ligation. All the mice used in the experiments were 9 weeks of age, following a 1 week acclimation period. The UUO-operated mice were randomly divided into the following groups ( $\mathrm{n}=5-7$; housed in groups): Sham, UUO-control and UUO-fasudil (1 g/l, Mitsubishi Tanabe Pharma Corporation). Fasudil was administered in the drinking water 2 days prior to the UUO surgery until the day of sacrifice. The mice were sacrificed under anesthesia with sevoflurane on days 3, 7 and 14 following surgery. The kidneys were then removed and divided into several parts for RNA, hydroxyproline and immunohistochemistry assays, and protein analysis.

Cell culture. The NRK-52E rat kidney tubular epithelial cell line, NRK-49F fibroblast cell line and RAW264.7 mouse macrophage cell line, obtained from American Type Culture Collection (Manassas, VA, USA), were cultured in low glucose $(5 \mathrm{mmol} / \mathrm{l})$ Dulbecco's modified Eagle's medium (cat. no. D6046; Sigma-Aldrich, St. Louis, MO, USA) supplemented with $10 \%$ fetal bovine serum (cat. no. S1820; Biowest, Nuaille, France) at $37^{\circ} \mathrm{C}$ in a $5 \% \mathrm{CO}_{2}$ atmosphere. For experimental treatments, the cells were seeded into 24-well plates in

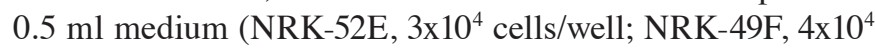
cells/well; RAW264.7, 2x10 5 cells/well) for the determination of mRNA expression levels. The cells were seeded into 12-well plates in $1.5 \mathrm{ml}$ medium (NRK-52E, 5x10 4 cell/well; NRK-49F, $6 \times 10^{4}$ cells/well) to determine the protein expression levels. After $24 \mathrm{~h}$, serum was reduced to $1 \%$ for $20 \mathrm{~h}$ at $37^{\circ} \mathrm{C}$, and recombinant human TGF- $\beta 1$ (10 ng/ml; cat. no. 100-21C; Peprotech, Rocky Hill, NJ, USA) or lipopolysaccharide (LPS; $500 \mathrm{ng} / \mathrm{ml}$; cat. no. L4391; Sigma-Aldrich) were added for $24 \mathrm{~h}$ at $37^{\circ} \mathrm{C}$. To investigate the role of ROCK under these conditions, 10 or $30 \mu \mathrm{mol} / 1$ hydroxyfasudil (Mitsubishi Tanabe Pharma Corporation), a bioactive metabolite of fasudil, was added to the cells $1 \mathrm{~h}$ prior to TGF- $\beta 1$ or LPS stimulation at $37^{\circ} \mathrm{C}$.

Histology and immunohistochemistry. The kidneys were removed and immediately fixed in $10 \%$ formaldehyde neutral buffer solution (Nacalai Tesque, Inc., Kyoto, Japan). The formalin-fixed kidneys were embedded in paraffin, and the paraffin sections were stained with hematoxylin (cat. no. 115938; Merck Millipore, Darmstadt, Germany) and eosin (cat. no. 115935; Merck Millipore) and Sirius Red (cat. no. 365548; Sigma-Aldrich)/Fast Green (cat. no. 069-00032; Wako Pure Chemical Industries, Ltd., Osaka, Japan). Immunohistochemistry was performed, as described previously (21). Briefly, the kidney paraffin sections ( $4 \mu \mathrm{m}$ thick) were deparaffinized, and the sections were pretreated with $3 \%$ hydrogen peroxide (Junsei Chemicals, Tokyo, Japan). For F4/80, the sections were processed for antigen retrieval using protease (cat. no. 415231; Nichirei Histofine; Nichirei Biosciences, Inc., Tokyo, Japan) treatment at $37^{\circ} \mathrm{C}$ for $10 \mathrm{~min}$. The sections were incubated overnight at $4^{\circ} \mathrm{C}$ with anti- $\alpha$-SMA mouse monoclonal antibody (1:500; cat. no. A2547; Sigma-Aldrich) or anti-F4/80 rat monoclonal antibody (1:100; cat. no. MCA497G; AbD Serotec, Kidlington, UK), followed by incubation with horseradish peroxidase (HRP)-conjugated anti-mouse IgG secondary antibody (cat. no. 414321; Histofine Mouse Stain kit; Nichirei Biosciences, Inc.) and HRP-conjugated anti-rat IgG secondary antibody (cat. no. 414311; Histofine Simple Stain Mouse MAX PO Rat reagent; Nichirei Biosciences, Inc.), respectively. The sections were then stained with 3,3'-diaminobenzidine (Nacalai Tesque, Inc.) and counterstained with hematoxylin (cat. no. 30002; Muto Pure Chemicals Co., Ltd., Tokyo, Japan). Images of the sections were captured using a DP73 digital camera system (Olympus Corporation, Tokyo, Japan) equipped with CellSens software (ver. 1.6; Olympus Corporation) and a BX51 microscope (Olympus Corporation). For image analysis, whole-slide digital images of the sections were obtained using an Aperio Scan Scope XT (Leica Microsystems, Wetzlar, Germany). The Sirius Red-, $\alpha$-SMA-, and F4/80-positive areas were determined using Image-Pro Plus software (ver. 6.1.0.372; Media Cybernetics, Bethesda, MD, USA), as described previously (21).

Determination of kidney hydroxyproline content. The collagen content in the kidney was determined using the hydroxyproline assay, as previously described $(22,23)$ with modifications. In brief, the kidneys were homogenized in phosphate-buffered saline (Gibco Life Technologies, Carlsbad, CA, USA) at $700 \mu \mathrm{l} / 100 \mathrm{mg}$ kidney weight, completely hydrolyzed in $6 \mathrm{~mol} / \mathrm{l} \mathrm{HCl}$ (Wako Pure Chemical Industries, Ltd.) at $120^{\circ} \mathrm{C}$ 
for $6 \mathrm{~h}$, and filtered through a $0.45 \mu \mathrm{m}$ Millex-HV filter (Merck Millipore). The samples were dried by vacuum centrifugation using EZ-2 Plus (Genevac, Suffolk, UK) for $16 \mathrm{~h}$. The dried samples were then solubilized in distilled water. The samples were oxidized using chloramine T solution, containing $1.4 \%$ sodium $p$-toluenesulfonchloramide trihydrate (chloramine $\mathrm{T}$; Wako Pure Chemical Industries, Ltd.) and 10\% n-propanol (Wako Pure Chemical Industries, Ltd.) in citric acid buffer, which consisted of $0.26 \mathrm{~mol} / \mathrm{l}$ citric acid (Sigma-Aldrich), $0.88 \mathrm{~mol} / \mathrm{l}$ sodium acetate trihydrate (Wako Pure Chemical Industries, Ltd.), $0.85 \mathrm{~mol} / \mathrm{l}$ sodium hydroxide (Wako Pure Chemical Industries, Ltd.) and $1.2 \%$ acetic acid (Kanto Chemical Co., Inc., Tokyo, Japan). Following incubation at room temperature for $20 \mathrm{~min}$, Ehrlich's solution, containing $1 \mathrm{~mol} / \mathrm{l}$ 4-dimethylaminobenzaldehyde (Sigma-Aldrich), $18 \%$ perchloric acid (Sigma-Aldrich) and 60\% $n$-propanol was added, and the samples were incubated at $65^{\circ} \mathrm{C}$ for $40 \mathrm{~min}$. Absorbance was measured at $560 \mathrm{~nm}$ (SpectraMax M5e; SoftMax Pro ver. 5.4.1; Molecular Devices, Sunnyvale, CA, USA). The concentration of hydroxyproline was estimated from a standard curve, which was prepared using a pure solution of $l$-hydroxyproline (Wako Pure Chemical Industries, Ltd.), with the final results expressed as hydroxyproline per $\mathrm{mg}$ protein. The kidney protein concentration was determined using a Bicinchoninic Acid (BCA) protein assay (Thermo Fisher Scientific, Waltham, MA, USA), with bovine serum albumin as a standard.

Reverse transcription-quantitative polymerase chain reaction (RT-qPCR) analyses. Total RNA was extracted from the kidney using TRIzol reagent (Invitrogen Life Technologies) and purified using an RNeasy Mini kit (cat. no. 74106; Qiagen, Venlo, Netherlands), according to the manufacturer's instructions. The total RNA concentration was determined using a NanoDrop 1000 Spectrophotometer (Thermo Fisher Scientific). cDNA was synthesized from $1 \mu \mathrm{g}$ of total RNA and the reagent of the Master Mix, which included enzymes, random primers and other reaction reagents (cat. no. 11755250; SuperScript VILO MasterMix; Invitrogen, Life Technologies), using an iCycler Themal Cycler (Bio-Rad Laboratories, Inc., Hercules, CA, USA) under the following conditions: $25^{\circ} \mathrm{C}$ for $10 \mathrm{~min}, 42^{\circ} \mathrm{C}$ for $60 \mathrm{~min}$ and $85^{\circ} \mathrm{C}$ for $5 \mathrm{~min}$. qPCR was performed using TaqMan technology. The TaqMan Gene Expression Assay reagents, containing the mixture of primers and TaqMan probe, were obtained from Applied Biosystems Life Technologies (Foster City, CA, USA), as follows: ROCK1 (cat. no. Mm00485745_m1), ROCK2 (cat. no. Mm00485761_m1), TGF- $\beta 1$ (cat. no. Mm00441724_ m1), $\alpha$-SMA (cat. nos. Mm01546133_m1 and Rn01759928_g1), collagen 1a2 (cat. nos. Mm00483888_m1 and Rn01526721_m1), collagen 3a1 (cat. no. Mm01254477 $\mathrm{m} 1$ ), fibronectin (cat. no. Mm01256744_m1), MCP-1 (cat. nos. Mm00441242_m1 and Rn00580555_m1), F4/80 (cat.no.Mm00802529_m1),IL-1ß (cat.no.Mm00434228_m1), TNF $\alpha$ (cat.no.Mm00443258_m),IL-6 (cat. no. Mm00446190_ m1) and 18SrRNA (cat. no. 4308329). The reaction was performed using $1 \mu \mathrm{l}$ of cDNA in $20 \mu \mathrm{l}$ of final volume under the following conditions: $50^{\circ} \mathrm{C}$ for $2 \mathrm{~min}, 95^{\circ} \mathrm{C}$ for $10 \mathrm{~min}$ and 40 cycles of $95^{\circ} \mathrm{C}$ for $15 \mathrm{sec}$ and $60^{\circ} \mathrm{C}$ for $1 \mathrm{~min}$. This was performed using a 7500 Fast Real-Time PCR system (Applied
Biosystems Life Technologies). Data were analyzed using the standard curve method, and the results for each gene were normalized to 18SrRNA (an internal control).

Western blot analyses. The kidney tissues and cultured cells were homogenized in a lysis buffer containing $50 \mathrm{mmol} / \mathrm{l}$ Tris- $\mathrm{HCl}$ (pH 8.0; Nacalai Tesque Co., Ltd.), 150 mmol/1 NaCl (Wako Pure Chemical Industries, Ltd.), $0.5 \%$ sodium deoxycholate (Wako Pure Chemical Industries, Ltd.), $0.1 \%$ sodium dodecyl sulfate (Bio-Rad Laboratories, Inc.), 1\% Triton X-100 (Sigma-Aldrich), 1x protease inhibitors (complete; cat. no. 11697498001; Roche Diagnostics, Basel, Switzerland) and 1x phosphatase inhibitors (cat. no. P2850; Sigma-Aldrich). Following centrifugation at $15,000 \mathrm{x}$ g for $20 \mathrm{~min}$ at $4^{\circ} \mathrm{C}$ (CF-15R; Hitachi, Tokyo, Japan), the supernatant was collected as the lysate. The lysates were denatured for $5 \mathrm{~min}$ by boiling. Protein concentration was determined using the BCA protein assay (Thermo Fisher Scientific) with bovine serum albumin as a standard. Equal quantities of the lysates were separated on a 4-15\% TGX Precast gel (Bio-Rad Laboratories, Inc.) and transferred onto a polyvinylidene difluoride membrane (Bio-Rad Laboratories, Inc.) using a Trans-Blot Turbo Transfer system (Bio-Rad Laboratories, Inc.). The blots were blocked in Starting Block T20 (PBS) Blocking buffer (Thermo Fisher Scientific) at room temperature for $1 \mathrm{~h}$. They were subsequently incubated overnight at $4^{\circ} \mathrm{C}$ with the following primary antibodies: Anti-chicken phosphorylated myosin phosphatase target subunit-1 (p-MYPT-1; Thr850) rabbit polyclonal antibody (1:1,000; cat. no. 36-003; Merck Millipore), anti-rabbit GAPDH mouse monoclonal antibody (1:500; cat. no. MAB374; Merck Millipore), anti-rat collagen type I rabbit polyclonal antibody (1:2,000; cat. no. ABT123; Merck Millipore), anti-human p-Smad3 (Ser423+Ser425) rabbit polyclonal antibody (1:2,000; cat. no. ab51451; Abcam, Cambridge, UK), anti-human $\alpha$-SMA rabbit polyclonal antibody (1:500; cat. no. 14395-1-AP; Proteintech, Chicago, IL, USA) in Can Get Signal solution (Toyobo, Osaka, Japan). The blots were then incubated with the following secondary polyclonal antibodies: ECL donkey anti-rabbit IgG, HRP-conjugated species-specific whole antibody (1:5,000, cat. no. NA934; GE Healthcare Life Sciences, Chalfont, UK) and ECL sheep anti-mouse IgG, HRP-conjugated species-specific whole antibody (1:5,000; cat. no. NA931; GE Healthcare Life Sciences) at room temperature for $1 \mathrm{~h}$. The blots were developed using enhanced chemiluminescence (cat. no. 54-71-00; LumiGLO Reserve Chemiluminescent Substrate kit, KPL, Inc., Gaithersburg, MD, USA). The signal intensities of the specific bands were detected using an LAS-3000 Luminescent Image Analyzer (Fujifilm, Tokyo, Japan) and analyzed using the Multi Gauge program (ver. 3.0; Fujifilm). For quantification, the signal intensities were normalized to GAPDH, which was loaded in each well.

Statistical analysis. All data are expressed as the mean \pm standard error of the mean. The comparisons between the sham- and UUO-operated control mice, the control and fasudil-treated mice, the non-stimulated and stimulated cells, and the stimulated cells and hydroxyfasudil-treated cells were performed using Student's t-test. Statistical analyses were performed using the SAS system (ver. 8.0.0; SAS Institute Inc., Cary, NC, USA) in the biometrics section, and $\mathrm{P}<0.05$ was considered to indicate a statistically significant difference. 


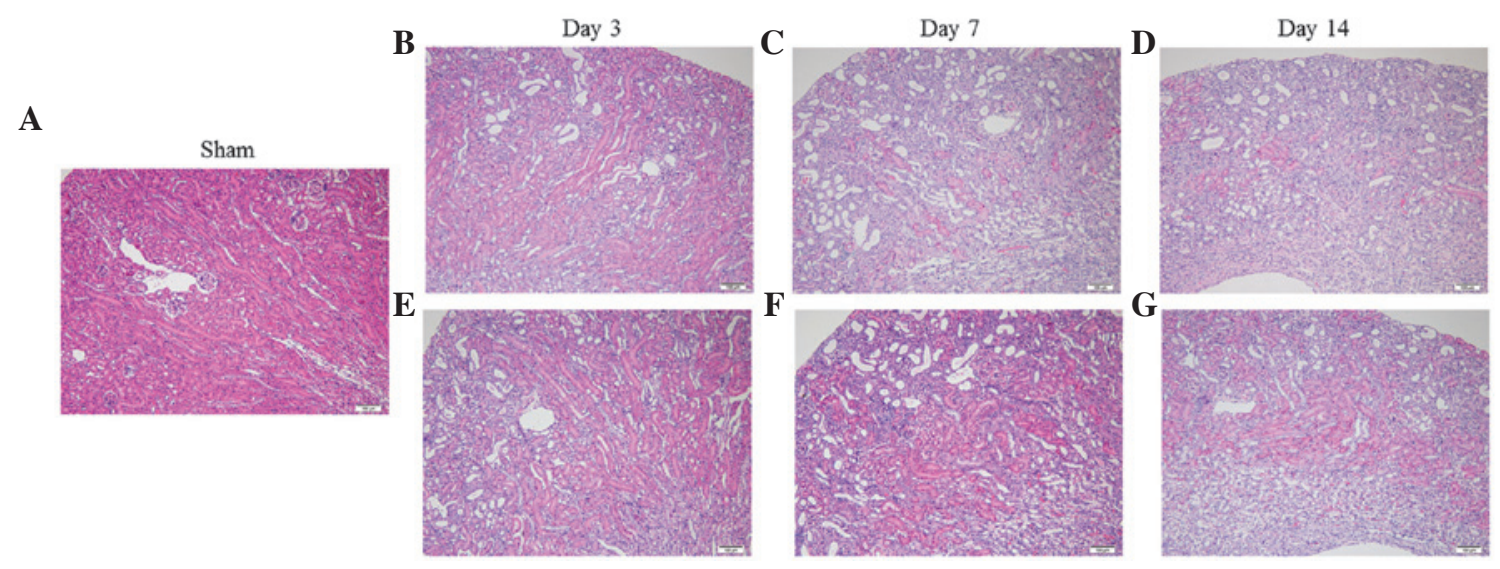

Figure 1. Histological changes in the kidney. Hematoxylin and eosin-stained sections of the sham-operated and UUO kidneys are shown. Representative sections from the (A) Sham group and the (B-D) UUO-control and (E-G) UUO-fasudil groups 3, 7 and 14 days following UUO (magnification, x10; scale bar=100 $\mu \mathrm{m}$ ). In the UUO kidneys, tubular atrophy and dilation were observed. UUO, unilateral ureteral obstruction.

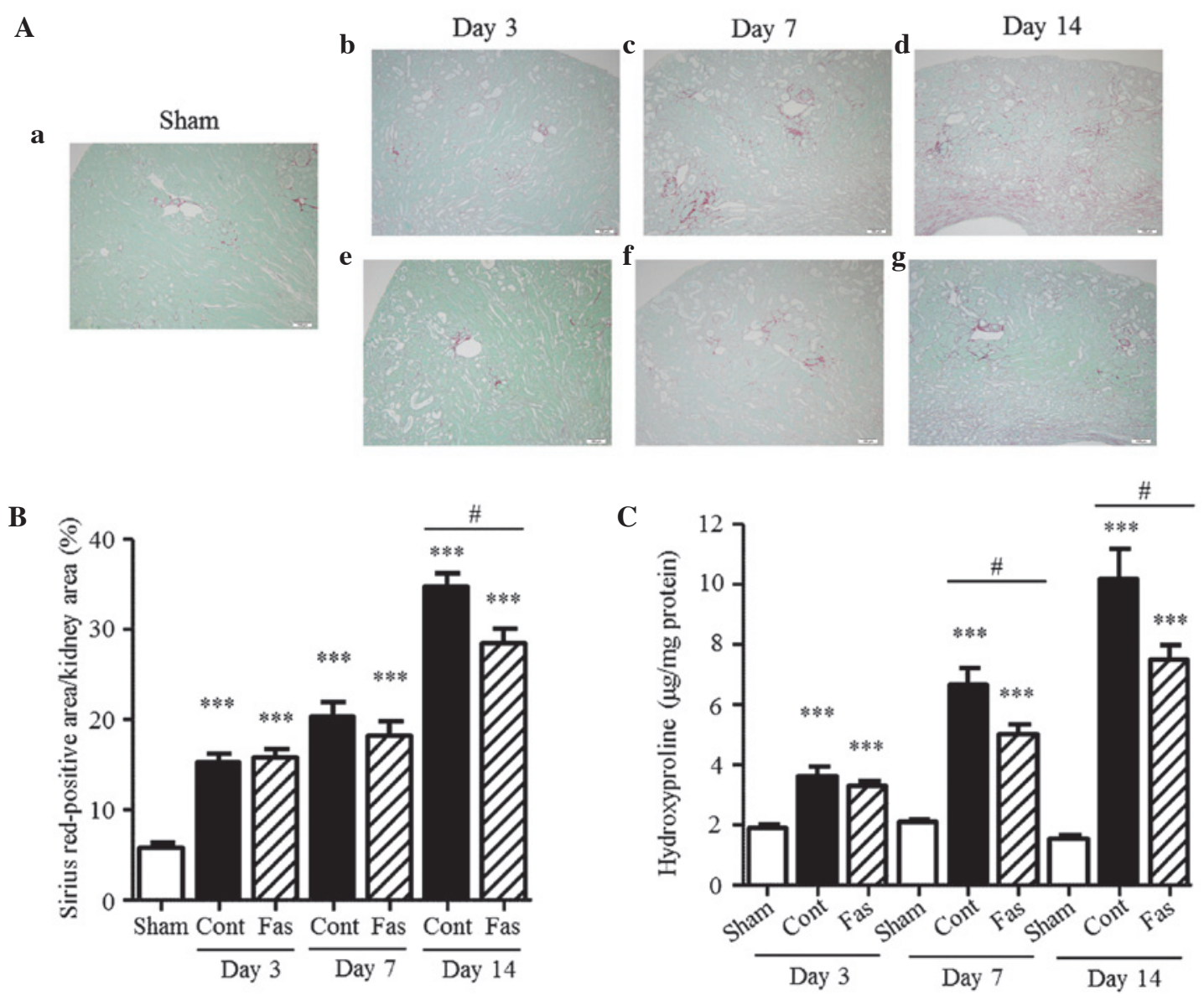

Figure 2. Sirius-Red staining and hydroxyproline content in the kidney. (A) Sirius Red-stained sections of the (a) sham-operated and UUO kidneys of the (b-d) Cont and (e-g) Fas groups 3, 7 and 14 days following UUO (magnification, x10; scale bar=100 $\mu \mathrm{m}$ ). Collagen deposition was stained red. (B) Sirius Red-positive area as a percentage of the total kidney area. (C) Hydroxyproline content in the sham-operated and UUO kidneys. The results are expressed as the mean \pm standard error of the mean $(\mathrm{n}=5-7) .{ }^{* * *} \mathrm{P}<0.001$, vs. sham group; ${ }^{*} \mathrm{P}<0.05$, vs. UUO-Cont at the same time-point. UUO, unilateral ureteral obstruction; Cont, control; Fas, fasudil.

\section{Results}

Effect of fasudil on renal histological changes. The kidneys of the UUO mice progressively developed renal parenchymal thinning, accompanied by severe tubulointerstitial damage, which included tubular atrophy/dilation, tubular regeneration and interstitial infiltration (Fig. 1), as reported previously $(24,25)$. Fasudil administration had minimal effect on these changes.

Fasudil attenuates interstitial fibrosis. To determine the effect of fasudil on interstitial fibrosis in the kidneys of the 
A

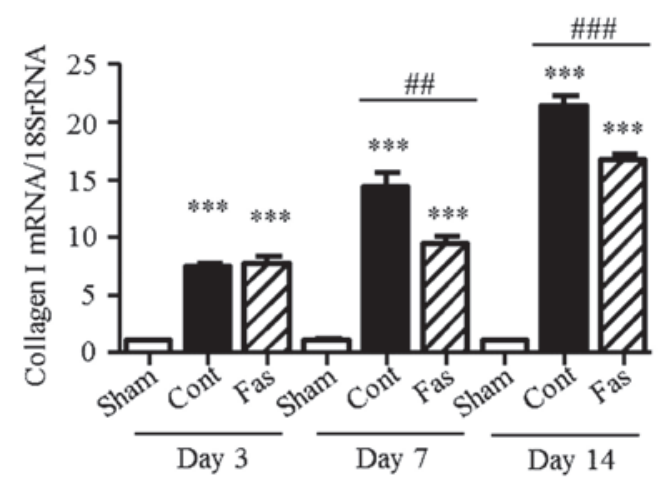

B

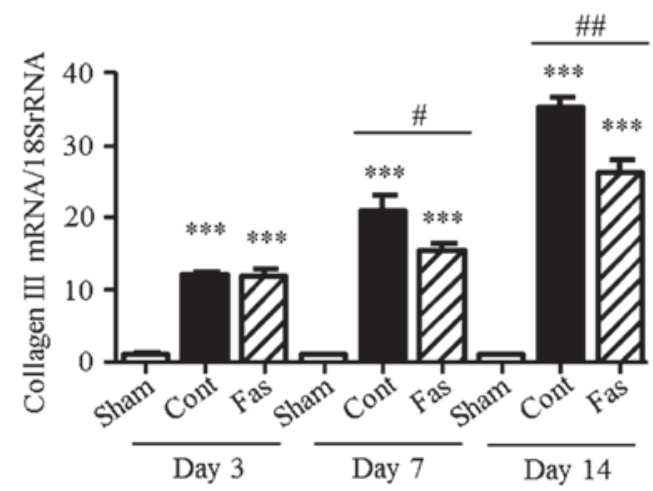

C

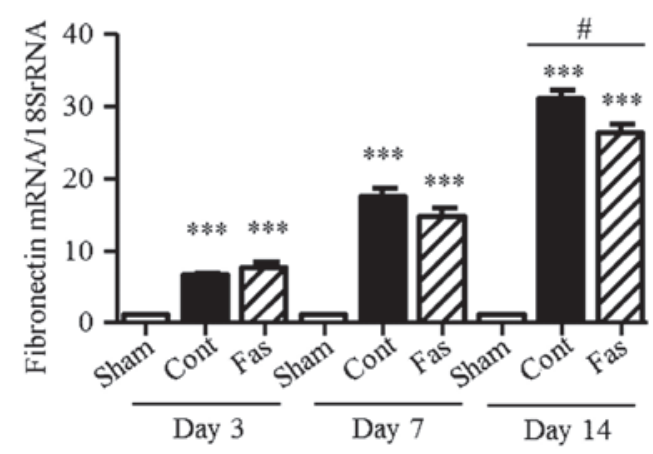

Figure 3. mRNA expression levels of collagen I, III and fibronectin in the kidney. The mRNA expression levels of (A) collagen I, (B) collagen III and (C) fibronectin in the sham-operated and UUO kidneys on days 3, 7 and 14 following UUO. The results are expressed as the mean \pm standard error of the mean $(\mathrm{n}=6-7)$. ${ }^{* * *} \mathrm{P}<0.001$, vs. sham group; ${ }^{~} \mathrm{P}<0.05,{ }^{\# \#} \mathrm{P}<0.01$ and ${ }^{\# \# \#} \mathrm{P}<0.001$, vs. UUO-Cont mice at the same time-points. UUO, unilateral ureteral obstruction; Cont, control; Fas, fasudil.

UUO mice, the collagen in the interstitium was stained with Sirius Red and the hydroxyproline content and extracellular matrix mRNA expression levels were measured. In the obstructed kidney, the Sirius Red-positive areas in the renal interstitium increased progressively, compared with those in the sham-operated kidney during the 14 day period (Fig. 2A and B), with 2.7-, 3.5- and 6.0-fold increases on days 3, 7 and 14 post-UUO, respectively. The content of hydroxyproline was also augmented, with 1.9-, 3.2- and 6.6-fold increases at day 3, 7 and 14 following UUO, respectively (Fig. 2C). Furthermore, the mRNA expression levels of collagen I, III and fibronectin were higher in the kidneys of the UUO mice, compared with those of the sham group, with collagen I levels 7.4-, 14.3- and 21.3-fold higher; collagen III levels 11.9-, 20.9- and 35.2-fold higher; and fibronectin levels 6.6-, 17.4- and 31.0-fold higher on days 3, 7 and 14 post-UUO, respectively (Fig. 3A-C). On day 14 following UUO, administration of fasudil significantly suppressed these augmented levels. On day 7 following UUO, the Sirius Red-positive areas and mRNA expression of fibronectin did not decrease significantly. However, the mRNA expression levels of collagen I and III, and the hydroxyproline content in the injured kidneys were markedly suppressed by fasudil administration. These results suggested that fasudil ameliorated the interstitial fibrosis.

Fasudil inhibits the expression of $\alpha$-SMA. The present study subsequently examined the effect of fasudil on interstitial myofibroblasts, characterized by the expression of $\alpha$-SMA. Immunohistochemical staining revealed increased numbers of $\alpha$-SMA-positive cells in the interstitium of the UUO mice, compared with the sham-operated mice, with 12.3-, 16.4- and 7.2-fold increases on days 3, 7 and 14 post-UUO, respectively (Fig. 4A and B). Similarly, the mRNA expression of $\alpha$-SMA increased in the UUO mice by 5.9-, 5.6- and 10.5-fold on days 3, 7, and 14 post-UUO, respectively (Fig. 4C). The immunohistochemical staining and mRNA expression analysis, which reduced 65 and 28\%, respectively, on day 7 post-UUO, demonstrated that fasudil administration markedly inhibited the increased expression of $\alpha$-SMA in the interstitium. Furthermore, the mRNA expression levels were significantly suppressed on day 14 following UUO (17\% reduction), compared with the control. These results indicated that fasudil administration inhibited the transformation of renal cells or extra-renal cells to myofibroblasts or the proliferation of myofibroblasts.

Effect of fasudil on macrophage infiltration. ROCK inhibitors have the ability to suppress cell migration (18-20). The present study examined the distribution of F4/80-positive cells and mRNA expression of F4/80 to assess the effect of fasudil on the macrophage infiltration to the kidney. The immunohistochemical staining detected F4/80-positive cells in the interstitium of the injured kidney; and their number in the UUO mice increased progressively, compared with the sham group, increasing 3.0-, 22.7-, and 30.2-fold on days 3, 7 and 14 post-UUO, respectively (Fig. 5A and B). Fasudil administration clearly inhibited macrophage infiltration into the injured kidney, with reductions of 55 and $28 \%$ on days 7 and 14 post-UUO, respectively. Similarly, the mRNA expression of F4/80 was increased 2.0-, 5.5- and 10.0-fold on days 3, 7, and 14 post UUO, respectively (Fig. 5C); and this increase was significantly inhibited by the administration of fasudil on day 14 following UUO. However, the augmented mRNA expression of MCP-1 was not affected by fasudil (Fig. 5D). These results suggested that fasudil inhibited the migration of macrophages to the injured kidney, but did not affect the expression of MCP-1.

Effect of fasudil on ROCK activity. To confirm the effect of fasudil on ROCK activity, the present study measured the phosphorylation of MYPT-1 in the kidney. The level of phosphorylation increased in the obstructed kidney, compared with the sham group (1.4-, 1.4- and 1.7-fold increase on days 3, 7 and 14 post-UUO, respectively; Fig. 6A). Fasudil treatment had no statistically significant effect on the phosphorylation levels; 
A

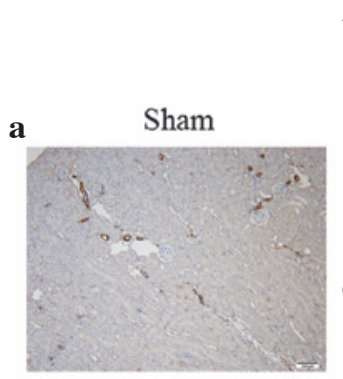

$\mathbf{B}$

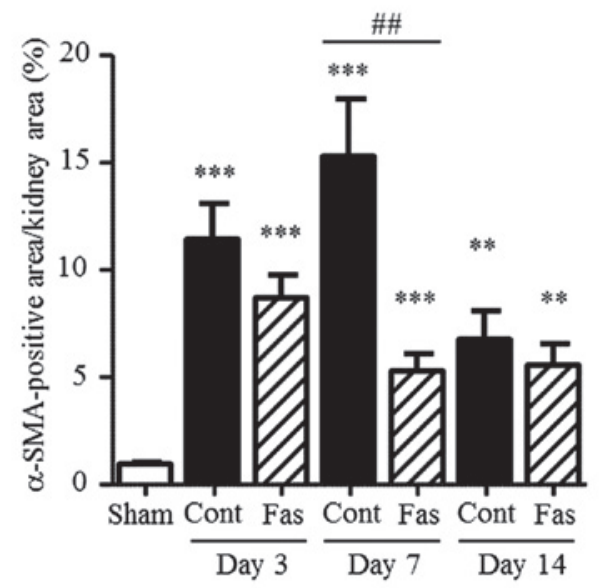

b

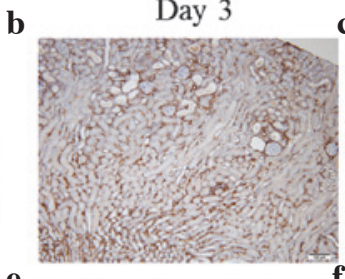

e

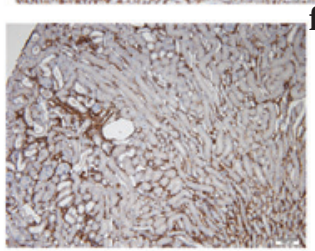

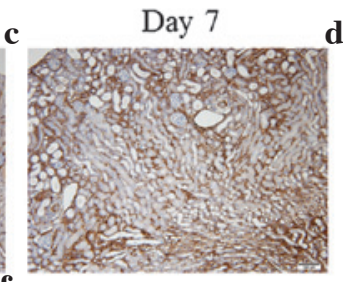

d Day 14

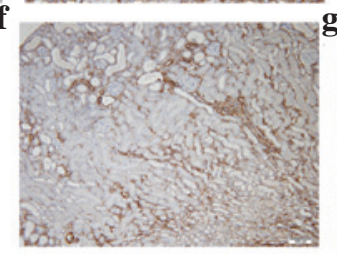

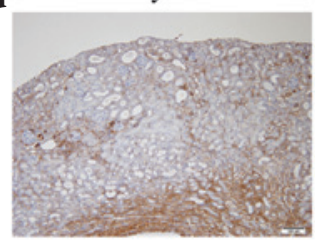

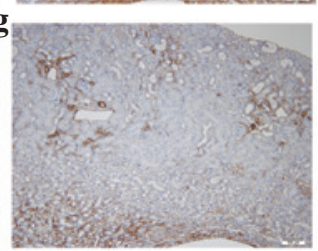

C

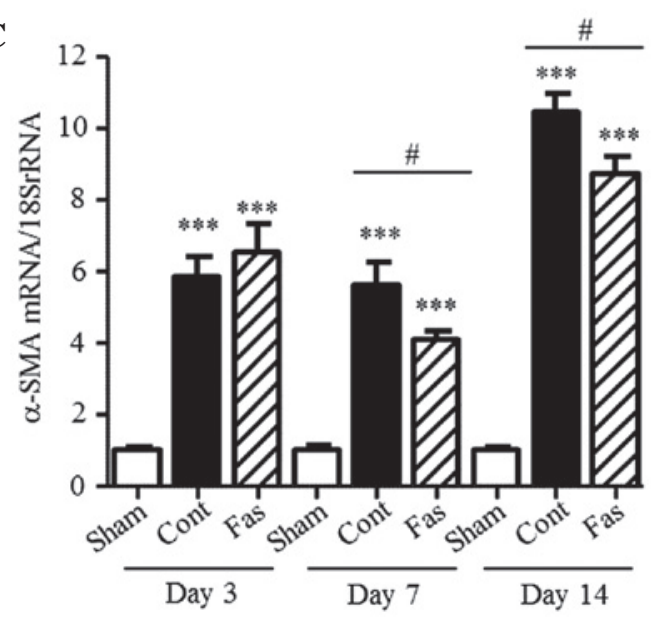

Figure 4. Expression of $\alpha$-SMA in the kidney. (A) $\alpha$-SMA immunohistochemistry of the (a) Sham, (b-d) UUO-Cont and (e-g) UUO-Fas groups on days 3 , 7, and 14 following UUO (magnification, x10; scale bar=100 $\mu \mathrm{m}$ ). $\alpha$-SMA-positive fibroblasts were stained brown. (B) $\alpha$-SMA-positive area as a percentage of the total kidney area. (C) mRNA expression of $\alpha$-SMA on days 3, 7 and 14 following UUO. The results are expressed as the mean \pm standard error of the mean $(\mathrm{n}=5-7)$. ${ }^{* * *} \mathrm{P}<0.01$ and ${ }^{* * * *} \mathrm{P}<0.001$, vs. sham group; ${ }^{*} \mathrm{P}<0.05$ and ${ }^{\# \#} \mathrm{P}<0.01$, vs. UUO-Cont mice at the same time-points. SMA, smooth muscle actin; UUO, unilateral ureteral obstruction; Cont, control; Fas, fasudil.

however, ROCK activity was suppressed to the basal level. The mRNA expression levels of ROCK 1 and ROCK 2 increased in the UUO mice, but fasudil did not affect their levels of expression (Fig. 6B and C).

Fasudil suppresses the TGF- $\beta$-Smad signaling pathway. It is known that the TGF- $\beta$-Smad signaling pathway is activated in the kidneys of UUO mice (26). To examine the effect of fasudil on this pathway, the present study measured the mRNA expression of TGF- $\beta 1$ and the level of $\mathrm{p}-\mathrm{Smad} 3$. The mRNA expression of TGF- $\beta 1$ increased in the UUO kidneys (3.0-, 6.0- and 7.2-fold on days 3, 7, and 14 post-UUO, respectively; Fig. 7A), compared with that in the sham-group. Fasudil administration significantly suppressed this increase in the obstructed kidney, by $18 \%$, on day 14 . In the kidneys of the UUO mice, the level of p-Smad3 progressively increased, compared with that in the sham-operated kidneys (3.8-, 5.3- and 13.1-fold on days 3, 7 and 14 post-UUO, respectively; Fig. 7B). This increase was markedly reduced by fasudil, by $29 \%$ on day 3 and $37 \%$ on day 7 following UUO. These results demonstrated that fasudil inhibited the TGF- $\beta$-Smad signaling pathway involved in interstitial fibrosis.

Effect of hydroxyfasudil on NRK-52E and NRK-49F cells. The results from the animal experiments indicated that fasudil attenuated renal interstitial fibrosis and the migration of macrophages to the kidney interstitium. Using NRK-52E and NRK-49F cells, the present study also investigated the effect of fasudil on the intrinsic renal cells. In the kidneys of UUO mice, TGF- $\beta 1$ was recognized as a vital mediator in renal fibrosis. In the NRK52-E and NRK-49F cells, TGF- $\beta 1$ induced the mRNA and protein expression levels of $\alpha$-SMA and collagen I (Fig. 8A and B). Hydroxyfasudil, a bioactive metabolite of fasudil, decreased the mRNA expression of $\alpha$-SMA mRNA induced by TGF- $\beta 1$ in the two cell types. Furthermore, in the NRK-49F cells, the protein expression of $\alpha$-SMA was markedly reduced following treatment with $30 \mu \mathrm{M}$ hydroxyfasudil. However, in the NRK-52E cells, no changes in the protein expression of $\alpha$-SMA were observed in this condition. In the NRK-49F cells, the increased mRNA expression of collagen I was significantly attenuated by $30 \mu \mathrm{M}$ hydroxyfasudil treatment; however, the protein expression was unaffected. By contrast, hydroxyfasudil did not affect the mRNA or protein expression levels of collagen I in the NRK-52E cells. In the kidneys of the UUO mice, the mRNA expression of MCP-1 increased, compared with the sham-operated group. These increased levels were unaffected by fasudil (Fig. 5D). The mRNA expression of MCP-1 was stimulated by TGF- $\beta 1$ in the NRK-52E and NRK-49F cells. Pretreatment with hydroxyfasudil clearly suppressed the induced mRNA 
A
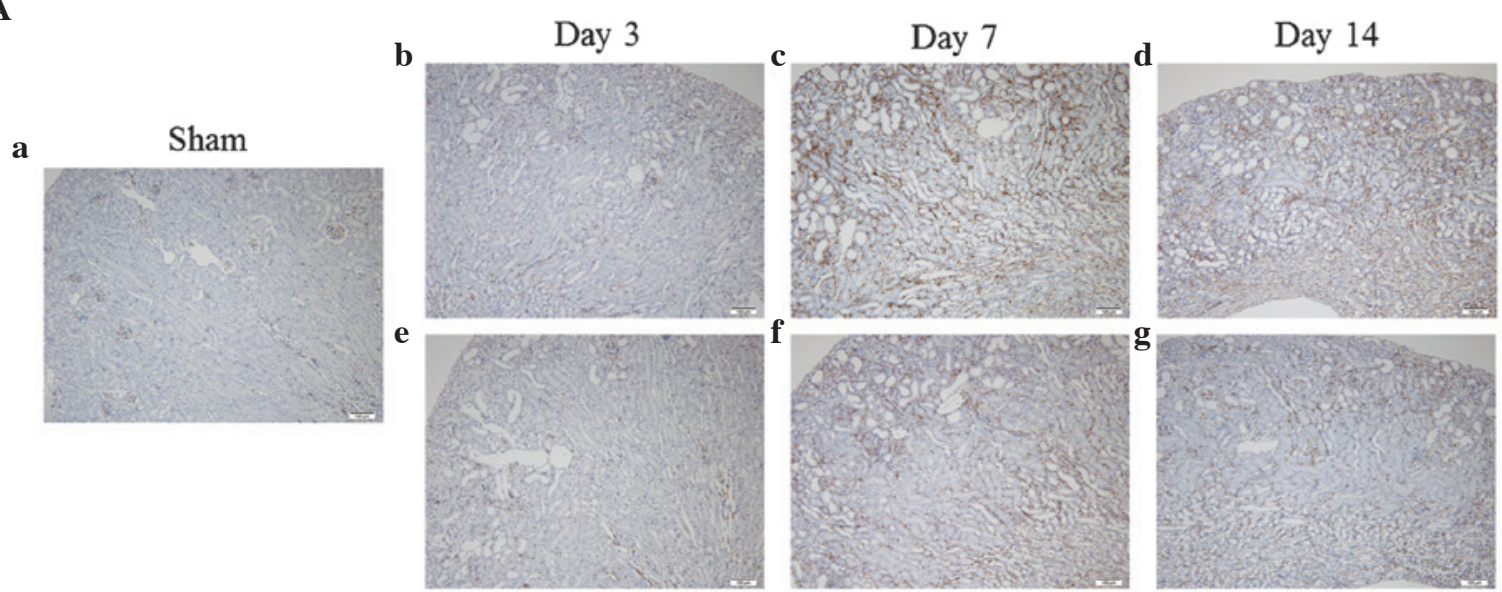

B

C
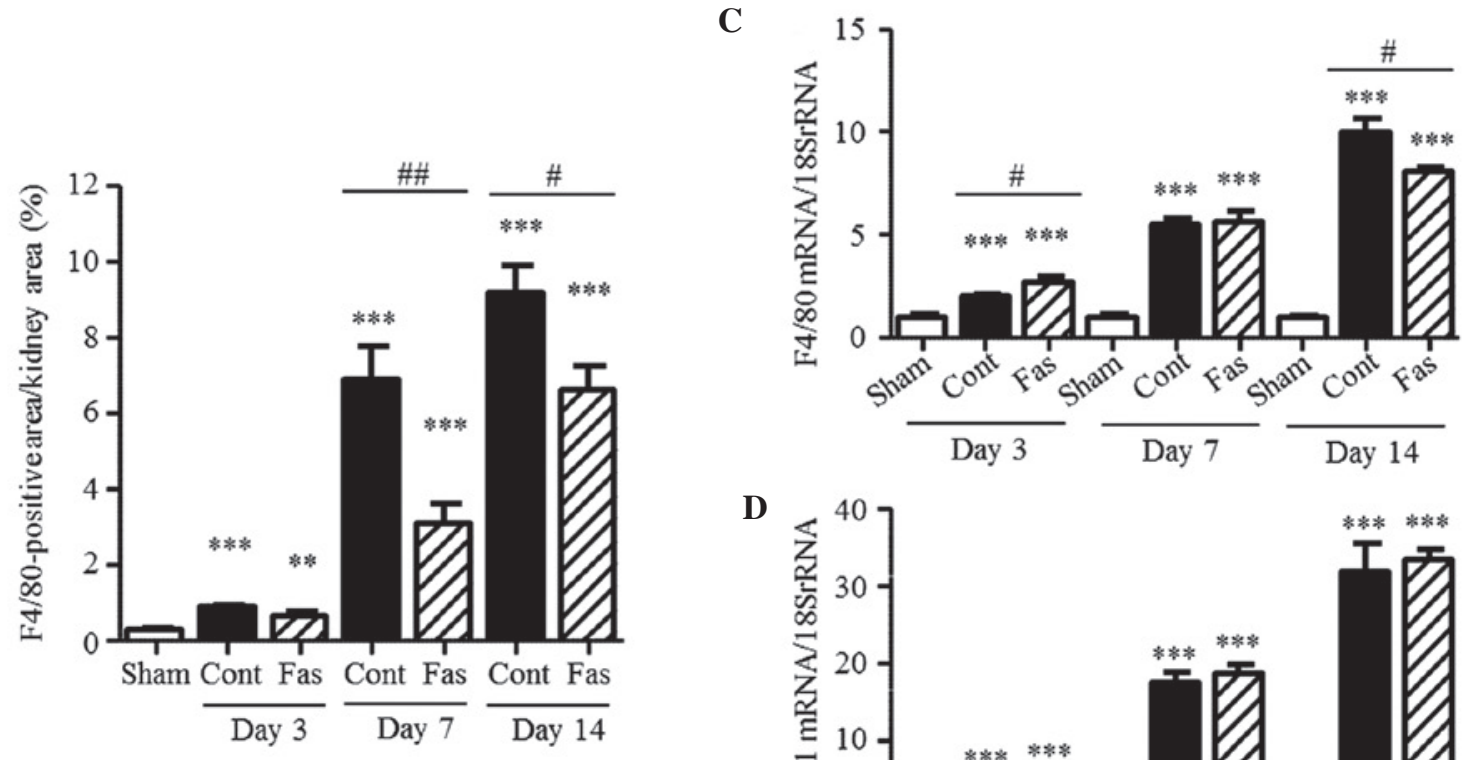

D

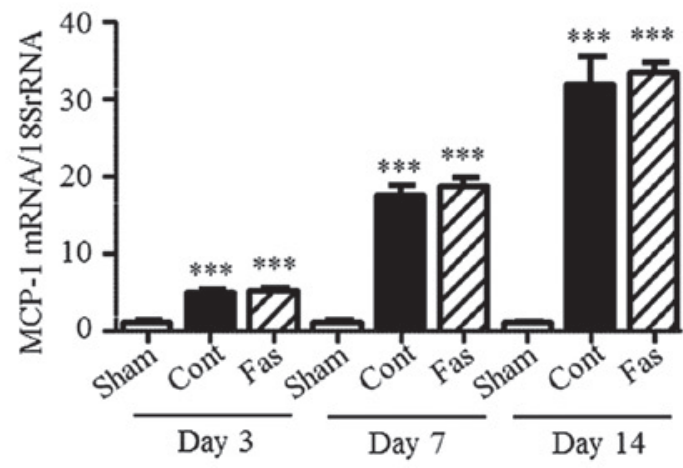

Figure 5. Expression levels of F4/80 and MCP-1 in the kidney. (A) F4/80 immunohistochemistry of the (a) sham-operated and kidneys in the (b-d) UUO-Cont and (e-g) UUO-Fas groups on days 3, 7 and 14 following UUO (magnification, x10; scale bars $=100 \mu \mathrm{m}$ ). F4/80-positive macrophages were stained brown. (B) F4/80-positive area as a percentage of the total kidney area. The mRNA expression levels of (C) F4/80 and (D) MCP-1 were determined in the sham-operated and UUO kidneys 3, 7 and 14 days following UUO. The results are expressed as the mean \pm standard error of the mean $(\mathrm{n}=5-7)$. ${ }^{* *} \mathrm{P}<0.01$ and ${ }^{* * *} \mathrm{P}<0.001$, vs. sham group; ${ }^{\#} \mathrm{P}<0.05$ and ${ }^{\# \#} \mathrm{P}<0.01$, vs. UUO-Cont mice at the same time-points. MCP-1, monocyte chemoattractant protein-1; UUO, unilateral ureteral obstruction; Cont, control; Fas, fasudil.
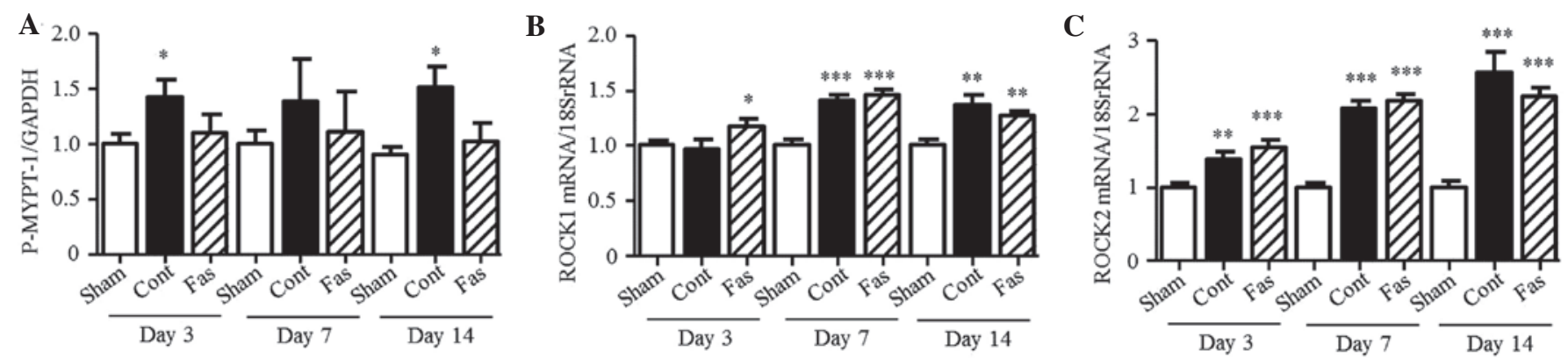

Figure 6. ROCK activity and the mRNA expression levels of ROCK1 and ROCK2 in the kidney. (A) Quantitative analysis revealing the ratio of relative abundance of p-MYPT-1/GAPDH proteins, detected using western blotting. The mRNA expression levels of (B) ROCK1 and (C) ROCK2 in the sham-operated and UUO kidneys 3, 7 and 14 days following UUO. The results are expressed as the mean \pm standard error of the mean $(\mathrm{n}=6-7) .{ }^{*} \mathrm{P}<0.05,{ }^{* *} \mathrm{P}<0.01$ and ${ }^{* * * *} \mathrm{P}<0.001$, vs. sham group. UUO, unilateral ureteral obstruction; Cont, control; Fas, fasudil. ROCK, Rho-associated coiled-coil kinase p-MYPT1, phosphorylated myosin phosphatase target subunit-1. 

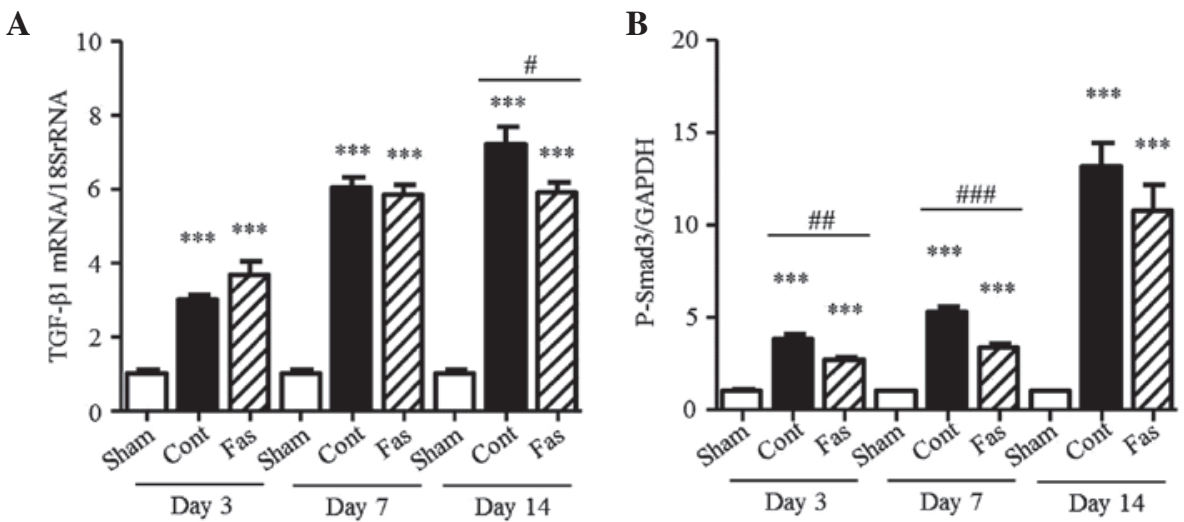

Figure 7. Expression levels of TGF- $\beta 1$ and p-Smad 3 in the kidneys. (A) mRNA expression levels of TGF- $\beta 1$ on days 3,7 and 14 following UUO. (B) Quantitative analysis revealing the ratio of the relative abundance of $\mathrm{p}-\mathrm{Smad} 3 / \mathrm{GAPDH}$ proteins, detected using western blot analysis. The results are expressed as the mean \pm standard error of the mean $(\mathrm{n}=6-7)$. ${ }^{* * *} \mathrm{P}<0.001$, vs. sham group; ${ }^{\#} \mathrm{P}<0.05,{ }^{\# \# /} \mathrm{P}<0.01$ and ${ }^{\# \# / t} \mathrm{P}<0.001$, vs. UUO-Cont mice at the same time-points. TGF, transforming growth factor; p-phosphorylated; UUO, unilateral ureteral obstruction; Cont, control; Fas, fasudil.
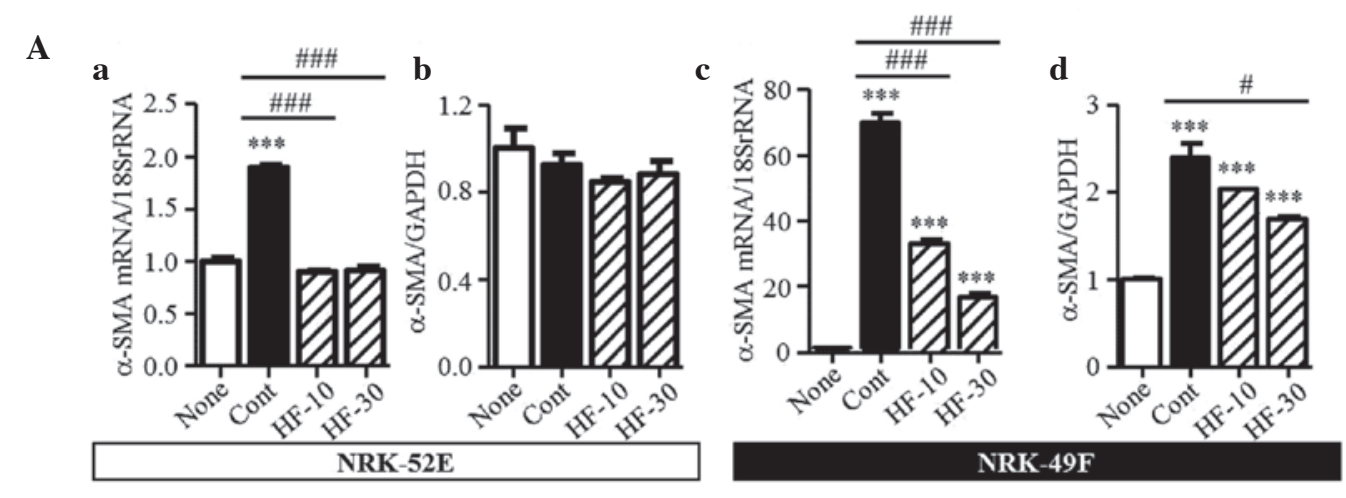

B

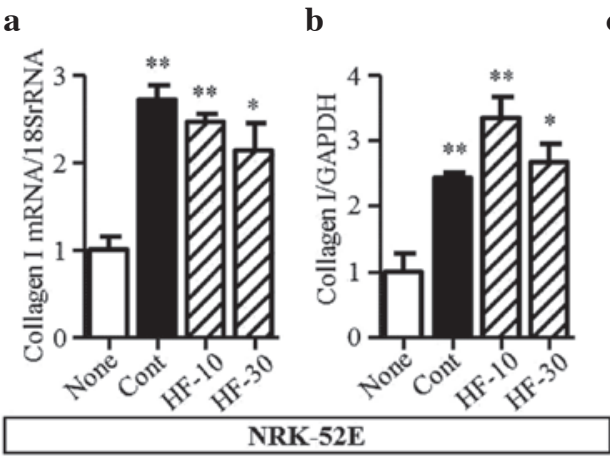

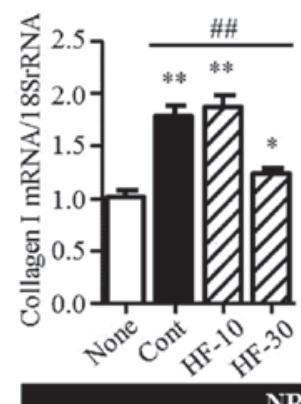

d

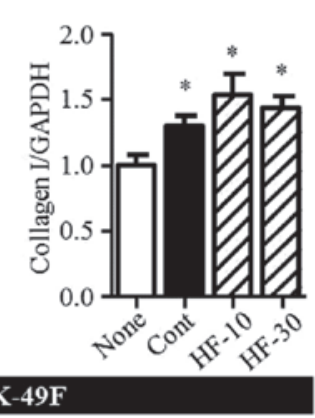

C



b

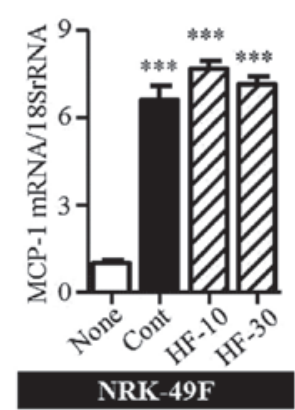

Figure 8. Expression levels of $\alpha$-SMA, collagen I and MCP-1 in the NRK-52E and NRK-49F cells. (Aa) mRNA and (Ab) protein expression levels of $\alpha$-SMA in the NRK-52E and the (Ac) mRNA and (Ad) protein expression levels of $\alpha$-SMA in the NRK-49F cells. mRNA and protein expression levels of collagen I in the (Ba and b) NRK-52E cells and the (Bc and d) NRK-49F cells. mRNA expression levels of MCP-1 in the (Ca) NRK-52E and (Cb) NRK-49F cells. Results are expressed as the mean \pm standard error of the mean $(\mathrm{n}=3) .{ }^{*} \mathrm{P}<0.05,{ }^{* *} \mathrm{P}<0.01$ and ${ }^{* * *} \mathrm{P}<0.001$, vs. none; ${ }^{\#} \mathrm{P}<0.05$, ${ }^{\# \#} \mathrm{P}<0.01$ and ${ }^{\# \# \#} \mathrm{P}<0.001$, vs. Cont. $\mathrm{SMA}$, smooth muscle actin; MCP-1, monocyte chemoattractant protein-1; None, no-stimulation; Cont, TGF- $\beta 1$ stimulation; HF-10 or HF-30, pretreatment with 10 or $30 \mu \mathrm{M}$ hydroxyfasudil prior to TGF- $\beta 1$ stimulation, respectively. 


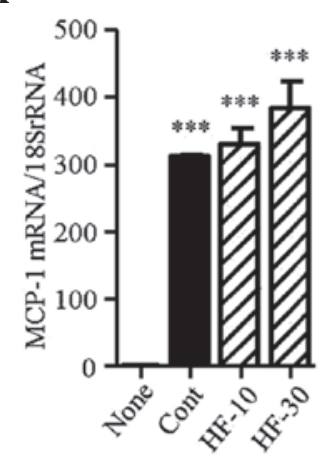

B

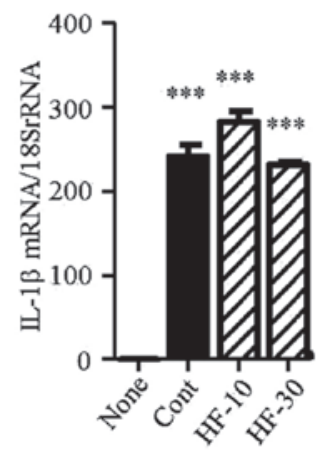

C

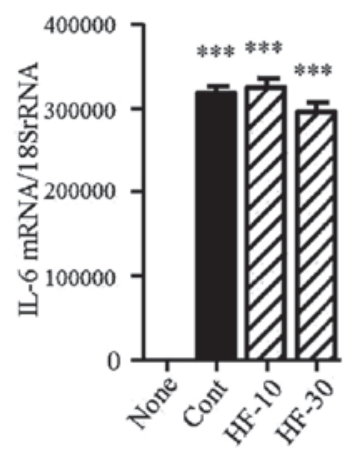

D



Figure 9. mRNA expression levels of chemokines/cytokines in RAW264.7 cells. The mRNA expression levels of (A) MCP-1, (B) IL-1 $\beta$, (C) IL-6 and (D) TNF 0 were determined in RAW264.7 cells. Results are expressed as the mean \pm standard error of the mean $(\mathrm{n}=3)$. ${ }^{*} \mathrm{P}<0.05$ and ${ }^{* * *} \mathrm{P}<0.001$, vs. none. MCP-1, monocyte chemoattractant protein-1; IL, interleukin; TNF, tumor necrosis factor; None, no-stimulation; Cont, lipopolysaccharide stimulation; HF-10 or HF-30, pretreatment with 10 or $30 \mu \mathrm{M}$ hydroxyfasudil prior to lipopolysaccharide stimulation, respectively.

expression of MCP-1 in the NRK-52E cells, but had no effect in the NRK-49F cells (Fig. 8C).

Effect of hydroxyfasudil on RAW264.7 cells. The migration of macrophages to the kidney interstitium was observed in the UUO model. At an early stage of UUO, M1 macrophages, which are the classically activated macrophages, infiltrate the kidney and are involved in inflammation and phagocytosis (27). In the present study, RAW264.7 cells were stimulated by LPS, as M1 subtype cells. In these activated RAW264.7 cells, the mRNA expression levels of MCP-1, IL-1 $\beta$, IL-6 and TNF $\alpha$ were markedly increased (Fig. 9A-D). Hydroxyfasudil did not affect the expression levels of these mRNAs.

\section{Discussion}

The UUO model is useful for examination of the mechanisms of tubulointerstitial fibrosis in vivo. Interstitial inflammation occurs rapidly (2-3 days) and the subsequent histological changes, including tubular dilation, tubular atrophy, and fibrosis are observed soon after ( $\sim 7$ days). These common changes are found in several tubulointerstitial diseases (25,28-31). A study involving ROCK1-knockout mice (26) reported no inhibition of interstitial fibrosis, although fasudil and Y27632 pharmacological inhibitors inhibit fibrosis in the UUO model (18-20). To clarify the mechanisms of suppression of the interstitial fibrosis, the present study investigated the inhibition of ROCK using the pharmacological ROCK inhibitor, fasudil.

The present study demonstrated that fasudil reduced renal interstitial fibrosis and attenuated histological changes in the UUO mice. The results of the immunohistochemical staining and mRNA expression analysis revealed that fasudil markedly suppressed the expression of $\alpha$-SMA in the injured kidney. In the process of the kidney fibrosis, the number of myofibroblasts producing extracellular matrix proteins increases in the tubular interstitium. These cells express a distinctive protein, $\alpha-S M A$ and, until recently, tubular epithelial cells have been considered the predominant source of the interstitial myofibroblasts. However, several studies have reported that transformation to myofibroblasts occurs in various cells, including epithelial cells, endothelial cells, bone marrow-derived cells, resident fibroblasts and pericytes (32-36). In the present study, the
NRK-52E tubular epithelial cell line, and NRK-49F fibroblast cell line were used to examine the transformation into myofibroblasts and analyze fibrosis-associated gene expression. In the NRK-49F cells, hydroxyfasudil attenuated the mRNA and protein expression levels of $\alpha$-SMA stimulated by TGF- $\beta 1$. The upregulated mRNA expression of collagen I was suppressed by high dose-hydroxyfasudil in the NRK-49F cells only. These results suggested that fasudil inhibited TGF- $\beta 1$-induced transformation and collagen synthesis in the renal fibroblast cells. In the NRK-52E cells, the mRNA expression levels of $\alpha$-SMA increased by TGF- $\beta 1$ stimulation and were suppressed by hydroxyfasudil pretreatment. However, the protein expression levels of $\alpha$-SMA were not affected by TGF- $\beta 1$ stimulation. It is possible that the basal expression level of $\alpha$-SMA is higher than that of other cells under the same conditions. Previous in vitro and in vivo studies have reported that, not only TGF- $\beta 1$, but the others, including angiotensin II, aldosterone and high glucose, induce collagen production in tubular epithelial cells or fibroblast cells, and ROCK inhibitors reduce collagen production $(16,37,38)$. In the NRK-52E and the NRK-49F cells, the mRNA and protein expression levels of collagen I were markedly increased by TGF- $\beta 1$ stimulation, but hydroxyfasudil did not suppress them enough. It is possible that stimuli other than TGF- $\beta 1$ induce the fibrotic responses in these cells. In the UUO mice, fasudil attenuated the TGF- $\beta 1$ mRNA expression and the activity of downstream, Smad3 phosphorylation. The results suggested that the inhibition of ROCK activity suppressed the TGF- $\beta$-Smad signaling pathway and subsequently induced fibrotic responses in tubular epithelial cells and renal fibroblast cells.

It has been reported that damaged tubular cells, interstitial myofibroblasts and macrophages produce cytokines, chemokines and growth factors in the UUO kidneys, triggering the accumulation of interstitial macrophages (39-41). In the present study, fasudil inhibited the infiltration of macrophages into the injured kidney. However, the mRNA expression of MCP-1 was not affected by fasudil administration. In the NRK-49F cells, hydroxyfasudil did not reduce the TGF- $\beta 1$-enhanced mRNA expression of MCP-1, however, in the NRK-52E cells, hydroxyfasudil markedly inhibited the increase in the mRNA expression of MCP-1 mRNA stimulated by TGF- $\beta 1$. Myofibroblasts in the injured kidney consist of several cell 
types (32-36) and it is reported that myofibroblasts derived from epithelial cells comprise a small population (34-36). In the present study, hydroxyfasudil inhibited the mRNA expression of MCP-1 in the epithelial NRK-52E cells. Thus, it is likely that fasudil is able to suppress the MCP-1 production in myofibroblasts that are derived from epithelial cells. However, these populations are small in comparison with other myofibroblast groups; therefore, fasudil may not be able to suppress the expression of MCP-1 in the overall myofibroblast cell population in the injured kidney. Several investigators have also reported that renal interstitial fibrosis can be reduced without inhibition of the mRNA expression of MCP-1 in UUO mice: however, the mechanism requires clarification (42-44). Thus, the precise association between MCP-1 and ROCK in the renal interstitial fibrosis process remains to be fully elucidated.

Macrophages are categorized into two functional phenotypes: Classically activated M1 and alternatively activated M2 $(45,46)$. In the first stage of UUO kidney injury, almost all the infiltrated macrophages exhibit the M1 phenotype (27). To examine the association between macrophages and ROCK in renal interstitial fibrosis, the present study assessed the LPS-induced inflammatory response in RAW264.7 cells. LPS stimulation elevated the mRNA expression levels of MCP-1, IL-1 $\beta$, IL-6, TNF $\alpha$, and iNOS, however, it was difficult to detect the mRNA expression levels of collagen and TGF- $\beta 1$ mRNA (data not shown), which were characteristic of the M2 phenotype. These gene expression patterns are characteristic for the phenotype of M1 macrophages (46). Fasudil treatment did not affect the expression of the genes induced by LPS in the RAW264.7 cells. These novel results suggested that ROCK may contribute to the infiltration of monocytes/macrophages into the interstitium of the injured kidney, rather than affect the expression of inflammatory cytokines/chemokines.

Taken together, the data of the present study suggested that the inhibition of ROCK suppressed renal interstitial fibrosis via the TGF- $\beta$-Smad signaling pathway and suppressed the infiltration of macrophages into the injured kidney. It is likely that ROCK contributes to the activation of the renal intrinsic cells and the migration of the extra-renal cells in the progression of renal interstitial fibrosis.

\section{Acknowledgements}

The authors would like to thank Dr Akiyoshi Fukamizu and Dr Junji Ishida (Life Science Center, Tsukuba Advanced Research Alliance, Tsukuba University, Ibaraki, Japan) for their guidance. The authors would also like to thank Dr Kenji Arakawa, Dr Rikako Yamauchi and Dr Taku Sato (Mitsubishi Tanabe Pharma Corporation) for supporting this investigation.

\section{References}

1. Satoh K, Fukumoto $\mathrm{Y}$ and Shimokawa H: Rho-kinase: Important new therapeutic target in cardiovascular diseases. Am J Physiol Heart Circ Physiol 301: H287-H296, 2011.

2. Hahmann C and Schroeter T: Rho-kinase inhibitors as therapeutics: From pan inhibition to isoform selectivity. Cell Mol Life Sci 67: 171-177, 2010.

3. Olson MF: Applications for ROCK kinase inhibition. Curr Opin Cell Biol 20: 242-248, 2008.
4. Ming D, Yan BP, Liao JK, Lam YY, Yip GW and Yu CM: Rho-kinase inhibition: A novel therapeutic target for the treatment of cardiovascular diseases. Drug Discov Today 15: 622-629, 2010.

5. Budzyn K, Marley PD and Sobey CG: Targeting Rho and Rho-kinase in the treatment of cardiovascular disease. Trends Pharmacol Sci 27: 97-104, 2006.

6. Shimokawa H and Rashid M: Development of Rho-kinase inhibitors for cardiovascular medicine. Trends Pharmacol Sci 28: 296-302, 2007.

7. Kushiyama T, Oda T, Yamamoto K, Higashi K, Watanabe A, Takechi H, Uchida T, Oshima N, Sakurai Y, Miura S and Kumagai H: Protective effects of Rho kinase inhibitor fasudil on rats with chronic kidney disease. Am J Physiol Renal Physiol 304: F1325-F1334, 2013.

8. Nishikimi T, Koshikawa S, Ishikawa Y, Akimoto K, Inaba C, Ishimura K, Ono H and Matsuoka H: Inhibition of Rho-kinase attenuates nephrosclerosis and improves survival in salt-loaded spontaneously hypertensive stroke-prone rats. J Hypertens 25: 1053-1063, 2007.

9. Kanda T, Wakino S, Hayashi K, Homma K, Ozawa Y and Saruta T: Effect of fasudil on Rho-kinase and nephropathy in subtotally nephrectomized spontaneously hypertensive rats. Kidney Int 64: 2009-2019, 2003.

10. Xie X, Peng J, Chang X, Huang K, Huang J, Wang S, Shen X, Liu $\mathrm{P}$ and Huang $\mathrm{H}$ : Activation of RhoA/ROCK regulates NF- $\mathrm{KB}$ signaling pathway in experimental diabetic nephropathy. Mol Cell Endocrinol 369: 86-97, 2013.

11. Zhou H, Li YJ, Wang M, Zhang LH, Guo BY, Zhao ZS, Meng FL, Deng YG and Wang RY: Involvement of RhoA/ROCK in myocardial fibrosis in a rat model of type 2 diabetes. Acta Pharmacol Sin 32: 999-1008, 2011.

12. Li Y, Zhu W, Tao J, Xin P, Liu M, Li J and Wei M: Fasudil protect the heart against ischemia-reperfusion injury by attenuating endoplasmic reticulum stress and modulating SERCA activity: The differential role for PI3K/Akt and JAK2/STAT3 signaling pathways. PLoS One 7: e48115, 2012.

13. Kentrup D, Reuter S, Schnöckel U, Grabner A, Edemir B, Pavenstädt H, Schober O, Schäfers $M$, Schlatter $E$ and Büssemaker E: Hydroxyfasudil-mediated inhibition of ROCK1 and ROCK 2 improves kidney function in rat renal acute ischemia-reperfusion injury. PLoS One 6: e26419, 2011.

14. Matoba K, Kawanami D, Okada R, Tsukamoto M, Kinoshita J, Ito T, Ishizawa S, Kanazawa Y, Yokota T, Murai N, et al: Rho-kinase inhibition privents the progression of diabetic nephropathy by downregulating hypoxia-inducible factor $1 \alpha$. Kidney Int 84: 545-554, 2013.

15. Diah S, Zhang GX, Nagai Y, Zhang W, Gang L, Kimura S, Hamid MR, Tamiya T, Nishiyama A and Hitomi H: Aldosterone induced mypfibroblastic transdifferentiation and collagen gene expression through the Rho-kinase dependent signaling pathway in rat mesangial cells. Exp Cell Res 314: 3654-3662, 2008.

16. Wei J, Li Z, Ma C, Zhan F, Wu W, Han H, Huang Y, Li W, Chen D and Peng Y: Rho kinase pathway is likely responsible for the profibrotic actions of aldosterone in renal epithelial cells via inducing epithelial-mesenchymal transition and extracellular matrix excretion. Cell Biol Int 37: 725-730, 2013.

17. Manickam N, Patel M, Griendling KK, Gorin Y and Barnes JL: RhoA/Rho kinase mediates TGF- $\beta 1$-induced kidney myofibroblast activation through Poldip2/Nox4-derived reactive oxygen species. Am J Physiol Renal Physiol 307: F159-F171, 2014.

18. Nagatoya K, Moriyama T, Kawada N, Takeji M, Oseto S, Murozono T, Ando A, Imai E and Hori M: Y-27632 prevents tubulointerstitial fibrosis in mouse kidneys with unilateral ureteral obstruction. Kidney Int 61: 1684-1695, 2002.

19. Satoh S, Yamaguchi T, Hitomi A, Sato N, Shiraiwa K, Ikegaki I, Asano T and Shimokawa H: Fasudil attenuates interstitial fibrosis in rat kidneys with unilateral ureteral obstruction. Eur J Pharmacol 455: 169-174, 2002.

20. Takeda Y, Nishikimi T, Akimono K, Matsuoka H and Ishimitsu T: Benefical effects of a combination of Rho-kinase inhibitor and ACE inhibitor on tubulointerstitial fibrosis induced by unilateral ureteral obstruction. Hypertens Res 33: 965-973, 2010.

21. Kakimoto T, Kimata H, Iwasaki S, Fukunari A and Utsumi H: Automated recognition and quantification of pancreatic islets in Zucker diabetic fatty rats treated with exendin-4.J Endocrinol 216: 13-20, 2013.

22. Woessner JF Jr: The determination of hydroxyproline in tissue and protein samples containing small proportions of this imino acid. Arch Biochem Biophys 93: 440-447, 1961. 
23. Kivirikko KI, Laitinen O and Prockop DJ: Modifications of a specific assay for hydroxyproline in urine. Anal Biochem 19: 249-255, 1967.

24. Chevalier RL, Forbes MS and Thornhill BA: Ureteral obstruction as a model of renal interstitial fibrosis and obstructive nephropathy. Kidney Int 75: 1145-1152, 2009.

25. Chevalier RL: Obstructive nephropathy: Towards biomarker discovery and gene therapy. Nat Clin Pract Nephrol 2: 157-168, 2006.

26. Shen B, Liu X, Fan Y and Qiu J: Macrophages regulate renal fibrosis through modulating TGF $\beta$ superfamily signaling. Inflammation 37: 2076-2084, 2014.

27. Yang HC, Zuo Y and Fogo AB: Models of chronic kidney disease. Drug Discov Today Dis Models 7: 13-19, 2010.

28. Löpez-Novoa JM, Martinez-Salgado C, Rodriguez-Peña AB and López-Hernández FJ: Common pathophysiological mechanisms of chronic kidney disease: Therapeutic perspectives. Pharmacol Ther 128: 61-81, 2010 .

29. Truong LD, Gaber L and Eknoyan G: Obstructive uropathy. Contrib Nephrol 169: 311-326, 2011.

30. Eddy AA, López-Guisa JM, Okamura DM and Yamaguchi I: Investigating mechanizes of chronic kidney disease in mouse models. Pediatr Nephrol 27: 1233-1247, 2012.

31. Fu P, Liu F, Su S, Wang W, Huang XR, Entman ML, Schwartz RJ Wei L and Lan HY: Signaling mechanism of renal fibrosis in unilateral ureteral obstructive kidney disease in ROCK1 knockout mice. J Am Soc Nephrol 17: 3105-3114, 2006.

32. Ballhause TM, Soldati R and Mertens PR: Sources of myofibroblats in kidney fibrosis: All answers are correct, however to different extent! Int Urol Nephrol 46: 659-664, 2014.

33. Duffield JS: Cellular and molecular mechanisms in kidney fibrosis. J Clin Invest 124: 2299-2306, 2014.

34. Pan SY, Chang YT and Lin SL: Microvascular pericytes in healthy and diseased kidneys. Int J Nephrol Renovascul Disease 7: 39-48, 2014.

35. LeBleu VS, Taduri G, O'Connell J, Teng Y, Cooke VG, Wada C, Sugimoto $\mathrm{H}$ and Kalluri R: Origin and function of myofibroblasts in kidney fibrosis. Nat Med 19: 1047-1053, 2013.
36. Jang HS, Kim JI, Jung KJ, Kim J, Han KH and Park KM: Bone marrow-derived cells play a major role in kidney fibrosis via proliferation and differentiation in the infiltrated site. Biochim Biophys Acta 1832: 817-825, 2013.

37. Qin J, Xie YY, Huang L, Yuan QJ, Mei WJ, Yuan XN, Hu GY, Cheng GJ, Tao LJ and Peng ZZ: Fluorofenidone inhibits nicotinamide adeninedinucleotide phosphate oxidase via PI3K/Akt pathway in the pathogenesis of renal interstitial fibrosis. Nephrology (Carlton) 18: 690-699, 2013.

38. Gu L, Gao Q, Ni L, Wang M and Shen F: Fasudil inhibits epithelial-myofibroblast transdifferentiation of human renal tubular epithelial HK-2 cells induced by high glucose. Chem Pharm Bull (Tokyo) 61: 688-694, 2013.

39. Gande MT, Pèrez-Barriocanal F and López-Novoa JM: Role of inflammation in tubule-interstitial damage associated to obstructive nephropathy. J Inflamm (Lond) 7: 19, 2010.

40. Wang Y and Harris DC: Macrophages in renal disease. J Am Soc Nephrol 22: 21-27, 2011.

41. Chung AC and Lan HY: Chemokines in renal injury. J Am Soc Nephrol 22: 802-809, 2011.

42. Liu N, Tolbert E, Pang M, Ponnusamy MI, Yan H and Zhuang S,: Suramin inhibits renal fibrosis in chronic kidney disease. J Am Soc Nephrol 22: 1064-1075, 2011.

43. Pang M, Ma L, Gong R, Tolbert E, Mao H, Ponnusamy M, Chin YE, Yan H, Dworkin LD and Zhuang S: A novel STAT3 inhibitor, S31-201, attenuates renal interstitial fibroblast activation and interstitial fibrosis in obstructive nephropathy. Kidney Int 78: 257-268, 2010.

44. Meng XM, Huang XR, Xiao J, Chen HY, Zhong X, Chung AC and Lan HY: Diverse roles of TGF- $\beta$ receptor II in renal fibrosis and inflammation in vivo and in vitro. J Pathol 227: 175-188, 2012.

45. Anders HJ and Ryu M: Renal microenvironments and macrophage phenotypes determine progression or resolution of renal inflammation and fibrosis. Kidney Int 80: 915-925, 2011.

46. Ricardo SD, van Goor H and Eddy AA: Macrophage diversity in renal injury and repair. J Clin Invest 118: 3522-3530, 2008. 